\title{
Metronidazole conjugated magnetic nanoparticles loaded with amphotericin B exhibited potent effects against pathogenic Acanthamoeba castellanii belonging to the T4 genotype
}

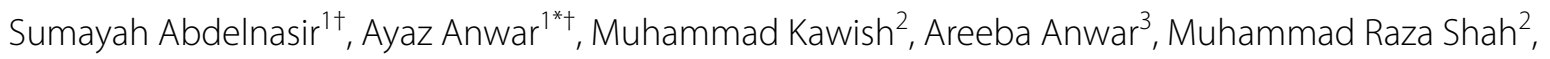
Ruqaiyyah Siddiqui ${ }^{4}$ and Naveed Ahmed Khan ${ }^{4^{*}}$ (D)

\begin{abstract}
Acanthamoeba castellanii can cause granulomatous amoebic encephalitis and Acanthamoeba keratitis. Currently, no single drug has been developed to effectively treat infections caused by Acanthamoeba. Recent studies have shown that drugs conjugated with nanoparticles exhibit potent in vitro antiamoebic activity against pathogenic free-living amoebae. In this study, we have developed a nano drug delivery system based on iron oxide nanoparticles conjugated with metronidazole which were further loaded with amphotericin B to produce enhanced antiamoebic effects against Acanthamoeba castellanii. The results showed that metronidazole-nanoparticles-amphotericin B (Met-MNPsAmp) significantly inhibited the viability of these amoebae as compared to the respective controls including drugs and nanoparticles alone. Met-MNPs-Amp exhibited $I C_{50}$ at $50 \mu \mathrm{g} / \mathrm{mL}$ against both $A$. castellanii trophozoites and cysts. Furthermore, these nanoparticles did not affect the viability of rat and human cells and showed safe hemolytic activity. Hence, the results obtained in this study have potential utility in drug development against infections caused by Acanthamoeba castellanii. A combination of drugs can lead to successful prognosis against these largely neglected infections. Future studies will determine the value of conjugating molecules with diagnostic and therapeutic potential to provide theranostic approaches against these serious infections.
\end{abstract}

Keywords: Magnetic nanoparticles, Metronidazole, Amphotericin B, Acanthamoeba

\footnotetext{
*Correspondence: ayazanwarkk@yahoo.com; naveed5438@gmail.com tSumayah Abdelnasir and Ayaz Anwar contributed equally to this manuscript

${ }^{1}$ Department of Biological Sciences, School of Science and Technology, Sunway University, Subang Jaya, 47500 Selangor, Malaysia

${ }^{4}$ Department of Biology, Chemistry and Environmental Sciences, College of Arts and Sciences, American University of Sharjah, Sharjah 26666, United Arab Emirates

Full list of author information is available at the end of the article
}

\begin{abstract}
Introduction
Nanoparticles (NPs) are proven future antimicrobials against bacteria, fungi, viruses, and parasites because of their broad activities against microbes including but not limited to the production of reactive oxygen species, DNA interaction, and metabolic functions (Hoseinzadeh et al. 2017; Vimbela et al. 2017; Wang et al. 2017). These characteristics of metallic nanoparticles improve the capacity of drug development against infectious diseases. Their physical properties including, size, surface charge, morphology and structure play pivotal roles in governing their antimicrobial potential (Ahmed et al.
\end{abstract}


2017; Anwar et al. 2019a, b, c). Metallic oxide nanoparticles including $\mathrm{ZnO}, \mathrm{CuO}, \mathrm{Fe}_{2} \mathrm{O}_{3}, \mathrm{NiO}$ and $\mathrm{MgO}$ etc. have shown potential in antimicrobial applications due to their easily modified surface functionalization with peptides, antibodies and therapeutic agents (Raghunath and Perumal 2017). Among metal oxide NPs, iron oxide NPs have been used most widely against various pathogenic bacteria due to potential bactericidal effects owing to their smaller size, magnetism, photothermal property and biocompatibility (Azam et al. 2012). However, to date, there are very few reports that account the affinity of any metal oxide nanoparticles against amoebae. In two recent reports, $\mathrm{TiO}_{2}$ nanoparticles have shown some potential against Acanthamoeba castellani triggered by external stimuli in both cases (Gomart et al. 2018; Imran et al. 2016a, b). Another report showed the limited efficacy of cobalt oxide $\left(\mathrm{Co}_{3} \mathrm{O}_{4}\right) \mathrm{NPs}$ as compared to cobalt phosphate $\left(\mathrm{Co}_{3}\left(\mathrm{PO}_{4}\right)_{2}\right)$ NPs against $A$. castellanii (Anwar et al. 2019a). Nanoparticle conjugation with broad spectrum antibiotics and natural products, however, has been found to be an effective strategy against brain-eating amoebae, particularly, N. fowleri (Anwar et al. 2019b, c; Rajendran et al. 2017).

Pathogenic free-living amoebae, including Naegleria fowleri, Balamuthia mandrillaris, and Acanthamoeba castellanii are opportunistic protists that cause infection of the central nervous system (CNS) (Visvesvara 2013; Schuster and Visvesvara 2004). Infection of the CNS by free-living amoebae almost always proves to be fatal (Visvesvara et al. 2007). This indicates the virulent nature of the pathogenic amoebae and the lack of effective treatment options against these parasites. The lack of awareness, lack of diagnostic modalities and lack of public knowledge about amoebae are considered to be one of many reasons of widespread amoebic infections worldwide (Ali et al. 2020). In addition, amoebae-related CNS infections are often misdiagnosed as other CNS infections, such as bacterial meningitis, due to the similarity in symptoms (Siddiqui and Khan 2014).

The blood-brain barrier (BBB) typically hampers the delivery of drugs into the CNS where the parasites usually reside. As a result, a higher dosage of drugs is required to treat this infection, which contributes to the severe side effects. Patients suffering from granulomatous amoebic encephalitis (GAE) are usually treated with multi-drug regimens which often include amphotericin $\mathrm{B}$, miltefosine and pentamidine isethionate but the prognosis of disease remains poor (Cope 2013).

The current study was aimed to develop metronidazole modified iron oxide nanoparticles loaded with amphotericin B (Met-MNPs-AmpB) and to test their antiamoebic potential against Acanthamoeba castellanii. We used AmpB as it shows limited effects against $A$. castellanii and the purpose of this study was to determine whether such a formulation would enhance its effects. The developed nanoparticles (Met-MNPs-AmpB) were thoroughly characterized through the determination of size, zeta potential, surface functional groups, and surface morphology using dynamic light scattering (DLS), Fourier transform infrared (FT-IR) spectroscopy and atomic force microscopy (AFM), respectively. The biocompatibility of MetMNPs-AmpB was evaluated on human cervical cancer cell lines (HeLa), as well as mouse embryonic cell lines (3T3 NIH) using 3-(4,5-dimethylthiazol-2-yl)-2,5-diphenyltetrazolium bromide (MTT) assay. Moreover, hemocompatibility analysis was also performed against fresh human erythrocytes. The antiamoebic properties of these nanoparticles were established against both trophozoite and cyst stages of $A$. castellanii in amoebicidal and cysticidal assays.

\section{Materials and methods Chemicals}

All organic solvents utilized in experiments were of highperformance liquid chromatography (HPLC) grade and purchased form Fisher scientific UK. Ferric Sulphatehexahydrate $\mathrm{Fe}_{2}\left(\mathrm{SO}_{4}\right)_{3} \cdot 6 \mathrm{H}_{2} \mathrm{O}$, Ferrous sulphateheptahydrate $\left(\mathrm{FeSO}_{4} \cdot 7 \mathrm{H}_{2} \mathrm{O}\right)$, 3-(Trimethoxysilyl)propyl methacrylate (MPTES), 4-dimethyl amino pyridine (DMAP), azobisisobutyronitrile (AIBN), ammonium hydroxide, dicyclohexylcarbodiimide (DCC), metronidazole and amphotericin B were obtained from Sigma-Aldrich, Merck Darmstadt (Germany) through a local supplier.

\section{Synthesis of Met-MNPs and Met-MNPs-Amp}

The synthesis of Met-MNPs-Ampwas carried out in multiple steps described in Fig. 1. First, compound 1 was synthesized by the following procedure. Methacrylic acid $(0.77 \mathrm{~g}, 9.0 \mathrm{mmol})$, 4-Dimethyl amino pyridine $(0.061 \mathrm{~g}$, $0.5 \mathrm{mmol}$ ) and dicyclohexylcarbodiimide (DCC, $1.85 \mathrm{~g}$, $9.0 \mathrm{mmol}$ ) were taken in a round bottom flask containing tetrahydrofuran (THF) $(20 \mathrm{~mL})$ connected with a condenser. The reaction was stirred for $10 \mathrm{~min}$ at $0{ }^{\circ} \mathrm{C}$ under Ar atmosphere. Metronidazole $(0.51 \mathrm{~g}, 3.0 \mathrm{mmol})$ was added later and stirred at $0{ }^{\circ} \mathrm{C}$ for $6 \mathrm{~h}$. The resulting mixture was concentrated in vacuo and then subjected to column chromatography using flash silica as a stationary phase. Compound 1 was obtained using hexane and ethyl acetate $(6: 4 \mathrm{v} / \mathrm{v})$ as mobile phases. Rf: 0.60 (DCM:MeOH, 9: 1, v/v). Yield 35\%; M.P.: $160-170{ }^{\circ} \mathrm{C}$, EI-MS m/z 239.1. ${ }^{1} \mathrm{H}$ NMR (300 MHz MeOD) $\delta$ ppm: 7.9 (s $1 \mathrm{H}$ imidazole), $2.4\left(\mathrm{~s} 3 \mathrm{H} \mathrm{CH}_{3}\right), 5.9(\mathrm{~s} 1 \mathrm{H} \mathrm{C}=\mathrm{C}), 5.5(\mathrm{~s} 1 \mathrm{H} \mathrm{C}=\mathrm{C}), 3.02(\mathrm{~s}$ $\left.3 \mathrm{H} \mathrm{CH}_{3}\right) 4.7\left(\mathrm{t} 2 \mathrm{H} \mathrm{CH}_{2}\right), 4.5\left(\mathrm{t} 2 \mathrm{H} \mathrm{CH}_{2}\right)$.

For the second step, a co-precipitation method was adopted for the preparation of narrow range MNPs first by using the previously described protocol (Petcharoen 
$\mathbf{a}$

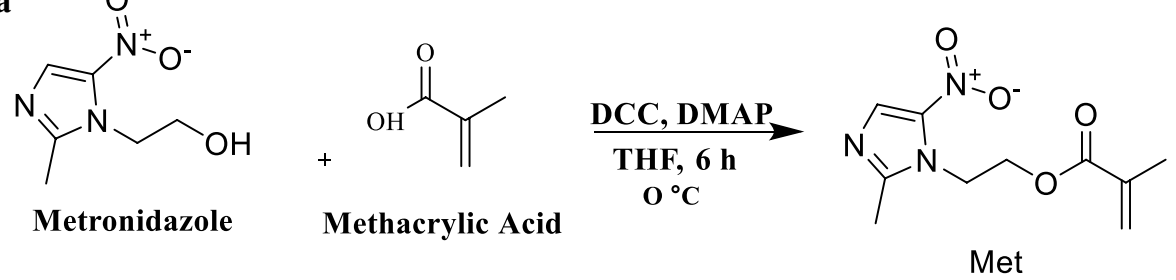

b
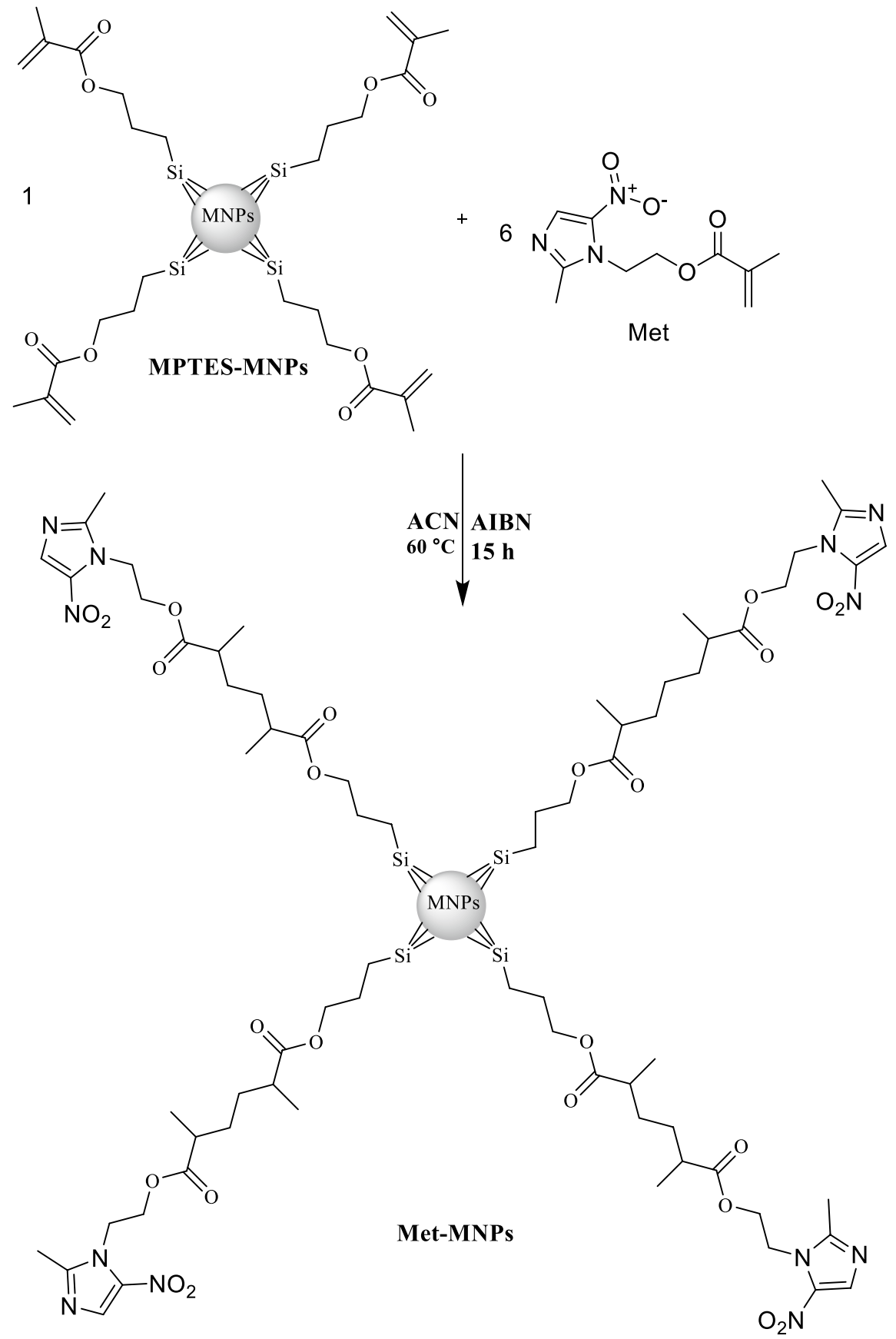

Fig. 1 a Synthetic scheme of derivative of metronidazole as Met. b Met-MNPs 
and Sirivat 2012). Here, MNPs was further etched with 3-(Trimethoxysilyl)propyl methacrylate (MPTES) through silanization reaction with slight modification (Saif et al. 2015). Briefly, a MNPs (10 mg/mL) suspension was prepared in ethanol and then MPTES was added in such a manner that the ratio between NPs to MPTES remains $1: 6$ and left with constant stirring at $60{ }^{\circ} \mathrm{C}$ for $4 \mathrm{~h}$. The resultant brownish suspension was washed several times with ethanol and freeze dried. Finally, polymerization was adopted with the aim to functionalize compound 1 on MPTES coated MNPs. Typically, MPTES-MNPs $(10 \mathrm{mg} / \mathrm{mL})$ dispersion was prepared in anhydrous ACN under Ar atmosphere. Then, compound $1(1.20 \mathrm{~g}, 5 \mathrm{mmol})$ was added to the above dispersion and after being stirred to $10 \mathrm{~min}$, AIBN $(1.32 \mathrm{~g}, 8 \mathrm{mmol})$ was added to the resulting mixture and refluxed for $15 \mathrm{~h}$ under Ar atmosphere at $60^{\circ} \mathrm{C}$. The prepared Met-MNPs underwent successive washing with $\mathrm{ACN}$ and were freeze dried. Met-MNPs were further exploited for their drug entrapment potential using a passive drug loading technique. Briefly, Met-MNPs were incubated with various equivalents of $\mathrm{AmpB}$ in methanol for $24 \mathrm{~h}$ on a shaker at $200 \mathrm{rpm}$ under ambient conditions. The resulting MetMNPs-Amp was removed by means of a permanent magnet and washed sequentially with water to remove the unloaded drug and stored at $4{ }^{\circ} \mathrm{C}$ for further analysis.

\section{Characterization of Met-MNPs-Amp Size, size distribution and morphology}

The average hydrodynamic diameter and polydispersity index (PDI) of vacant and Met-MNPs-Amp were investigated via Zetasizer (Zetasizer Nano ZS90 Malvern Instruments, Malvern, UK). Concisely diluted nanoparticles were cautiously transferred to a transparent plastic cuvette to avoid any bubble formation. The cuvette was then placed in the cell holder of the instrument and analysis was taken at $90^{\circ}$ scattering at $25^{\circ} \mathrm{C}$. The medium viscosity and refractive index were constant and kept at 1.0, 1.33 and $80.4 \mathrm{mPa}$, respectively. Nanoparticles were also characterized for morphology using atomic force microscopy (AFM, Agilent 5500). A drop of the formulation was placed on a mica slide and air dried at ambient temperature and placed under a microscope. The morphology was investigated at non-contact model.

\section{Drug entrapment efficiency determination}

Entrapment efficiency is described as the amount of drug entrapped into a carrier with respect to the initial amount of drug added (Manatunga et al. 2017). Therefore, the entrapment efficiency was determined by measuring the amount of unloaded drug at $405 \mathrm{~nm}$ by UV spectroscopy (Shimadzu 1800 series, Shimadzu Japan). Drug entrapment was investigated inthe following relation.

$$
\% \mathrm{EE}=\left(\mathrm{Q}_{\mathrm{t}}-\mathrm{Q}_{\mathrm{p}}\right) / \mathrm{Q}_{\mathrm{t}} \times 100
$$

$\mathrm{Q}_{\mathrm{p}}$ : Quantity of free drug, $\mathrm{Q}_{\mathrm{t}}$ : Quantity of drug added, \% EE: Entrapment efficiency of loaded drug in percent.

\section{Fourier Transformed Infrared (FT-IR) Spectroscopy}

Fourier transformed infrared (FT-IR, IR-470 spectrometer (Shimadzu, Kyoto, Japan)) analysis was performed in order to elucidate the possible drug entrapment and surface functionalization. Small amounts of powdered nanoparticles were mixed with $\mathrm{KBr}$ and subjected to a high pressure of 200 Psi to obtain self-supporting disks.

\section{Biocompatibility studies Hemocompatibility study}

Human blood was obtained from healthy individuals in the University of Karachi, Pakistan following relevant guidelines and regulations (Ethics committee approval ICCBS/IEC/LET/015/2018). Ethylenediaminetetraacetic acid (EDTA) stabilized fresh human blood samples $(5.0 \mathrm{~mL})$ were added to $10 \mathrm{~mL}$ of phosphate-buffered saline (PBS). Then, red blood cells (RBCs) were isolated via centrifugation at $6000 \mathrm{rpm}$ and washed several times with PBS solution. The purified RBCs were further diluted in $50 \mathrm{~mL}$ PBS and Triton $\mathrm{X}$ was used as the positive control, respectively. Then, $0.2 \mathrm{~mL}$ of diluted RBC suspension and $0.8 \mathrm{~mL}$ of Met-MNPs solutions in a range of $200-1000 \mu \mathrm{g} / \mathrm{mL}$ were mixed by vortexing. All sample tubes were kept in static condition at room temperature for $3 \mathrm{~h}$. Finally, the mixtures were centrifuged at $12,000 \mathrm{rpm}$ for $10 \mathrm{~min}$, and $1.5 \mathrm{~mL}$ of the supernatant of each sample was transferred to a cuvette. The absorbance values of the supernatants at $540 \mathrm{~nm}$ were determined by UV-Vis spectrophotometry. The percent hemolytic activity of RBCs was calculated using the following relation.

$$
\% \text { H.A }=R_{s} / R_{c} \times 100
$$

$\mathrm{R}_{\mathrm{s}}$ : Absorbance of sample, $\mathrm{R}_{\mathrm{c}}$ : Absorbance of positive control, \% H.A: Hemolytic activity in percent.

\section{In vitro cytotoxicity}

The synthesized nano carrier was screened for its cytotoxicity using MTT assay. Human cervical adenocarcinoma cells (HeLa) (ATCC ${ }^{\circledR} \mathrm{CCL}^{\mathrm{TM}}{ }^{\mathrm{TM}}$ ), were obtained from American Type Culture Collection (ATCC), and cultured in Roswell Park Memorial Institute (RPMI) 1640 medium, supplemented with $10 \%$ foetal bovine serum, $1 \%$ minimum essential medium amino acids, $1 \%$ L-glutamine and $1 \%$ antibiotics at $37{ }^{\circ} \mathrm{C}$ with $5 \% \mathrm{CO}_{2}$ (Rajendran et al. 2019). NIH 3T3 cells (ATCC CRL-1658) were purchased from the ATCC and cultured in Dulbecco's modified eagle`s medium (DMEM) having foetal bovine 
serum (10\%) (Invitrogen, USA) and antibiotics (streptomycin and penicillin-about $50 \mathrm{U} / \mathrm{mL}$ ). Both cell lines were incubated into well plates with 96 wells and $8 \times 10^{3}$ and $6 \times 10^{4}$ cells/well thickness, individually, in a $(200$ $\mu \mathrm{L})$ refined media. After incubation for about $24 \mathrm{~h}$, fresh media was introduced $(200 \mu \mathrm{L})$ consisting of Met-MNPs at various concentrations from 25 to $100 \mu \mathrm{g} / \mathrm{mL}$. Cells incubated in media without NPs were used as the negative control and developed for 48 h. 3-(4,5-Dimethylthiazol-2-Yl)-2,5-Diphenyltetrazolium Bromide solution $(\mathrm{MTT})$ in PBS was introduced into each well $(20 \mu \mathrm{L}$; $5 \mathrm{mg} / \mathrm{mL}$ ). The unreacted solution was expelled after $4 \mathrm{~h}$ incubation. The resulting formazan crystals were dissolved and introduced to $200 \mu \mathrm{L}$ DMSO per well before being analysed at $570 \mathrm{~nm}$ in a microplate reader. For the positive control and reference, standard Doxorubicin and Cyclohexylamine were used. The following relation was utilized to calculate the $\%$ cell viability.

$$
\text { Cell viability }=A_{t} / A_{c} \times 100
$$

$A_{t}$ : Mean of Absorbance value of Test Sample, $A_{c}$ : Mean of Absorbance value of Control.

\section{Culture of amoebae}

Acanthamoeba castellanii of T4 genotype (ATCC 50492) was purchased from American Tissue Culture Collection (ATCC). A. castellanii was cultured in growth medium consisting of Proteose peptone $(0.75 \% \mathrm{w} / \mathrm{v})$, yeast extract $(0.75 \% \mathrm{w} / \mathrm{v})$, and D-glucose $(1.5 \% \mathrm{w} / \mathrm{v})$ (PYG) (Anwar et al. 2019b).

\section{Amoebicidal assays}

Amoebicidal assays were performed as previously described (Rajendran et al. 2019). Briefly, $5 \times 10^{5}$ amoebae were incubated with compounds at various concentrations for $24 \mathrm{~h}$ at $30{ }^{\circ} \mathrm{C}$. The positive control used was chlorhexidine, respective solvents were used as solvent controls and RPMI-1640 alone was used as the negative control. A haemocytometer and $0.1 \%$ trypan blue solution were used to distinguish between live (unstained) and dead (stained) cells, in trypan blue exclusion assays. The number of viable amoebae determined were represented as \% cell death for graphical illustration.

\section{Cysticidal assays}

Acanthamoeba castellanii cysts were prepared by methods described previously (Anwar et al. 2019c). After the formation of mature cysts, $5 \times 10^{5}$ cysts were treated with various concentrations of Met-MNPs-Amp in 24-well plates in the presence of RPMI-1640 and incubated for $72 \mathrm{~h}$ at $30^{\circ} \mathrm{C}$. Chlorhexidine was used against $A$. castellanii as the positive control. RPMI-1640 alone was used as the negative control. After the incubation, the remaining viable cysts were enumerated using a hemocytometer by trypan blue exclusion method.

\section{Results}

The synthesis of the compound 1 (2-(2-methyl-5-nitro$1 H$-imidazol-1-yl)ethyl methacrylate) was achieved using carbodiimide coupling reaction (Fig. 1). The EI-MS spectrum showed $\mathrm{M}^{+}$peak at $\mathrm{m} / \mathrm{z} 239.1$ which coincides with the theoretical weight of the compound with molecular formula $\mathrm{C}_{10} \mathrm{H}_{13} \mathrm{~N}_{3} \mathrm{O}_{4}$ (Fig. 2a), respectively. The ${ }^{1} \mathrm{H}$ NMR spectra of the synthesized compound 1 shows aromatic singlet around $\delta 7.9 \mathrm{ppm}$ of $1 \mathrm{H}$ (Fig. 2b). Two singlets around $\delta 2.4 \mathrm{ppm}$ and $\delta 1.8 \mathrm{ppm}$ of $3 \mathrm{H}$ correspond to methyl group. Two singlets at $\delta 5.9 \mathrm{ppm}$ and $5.6 \mathrm{ppm}$ correspond to olefins. Two triplets at $\delta 4.7$ and $4.5 \mathrm{ppm}$ of $2 \mathrm{H}$ corresponds to $\mathrm{CH}_{2}$ of metronidazole. The reaction yielded $35 \%$ product. FT-IR analysis shows the coating of Met functional moieties onto the surface of MPTES NPs (Fig. 3a) and further AmpB loading (Fig. 3b).
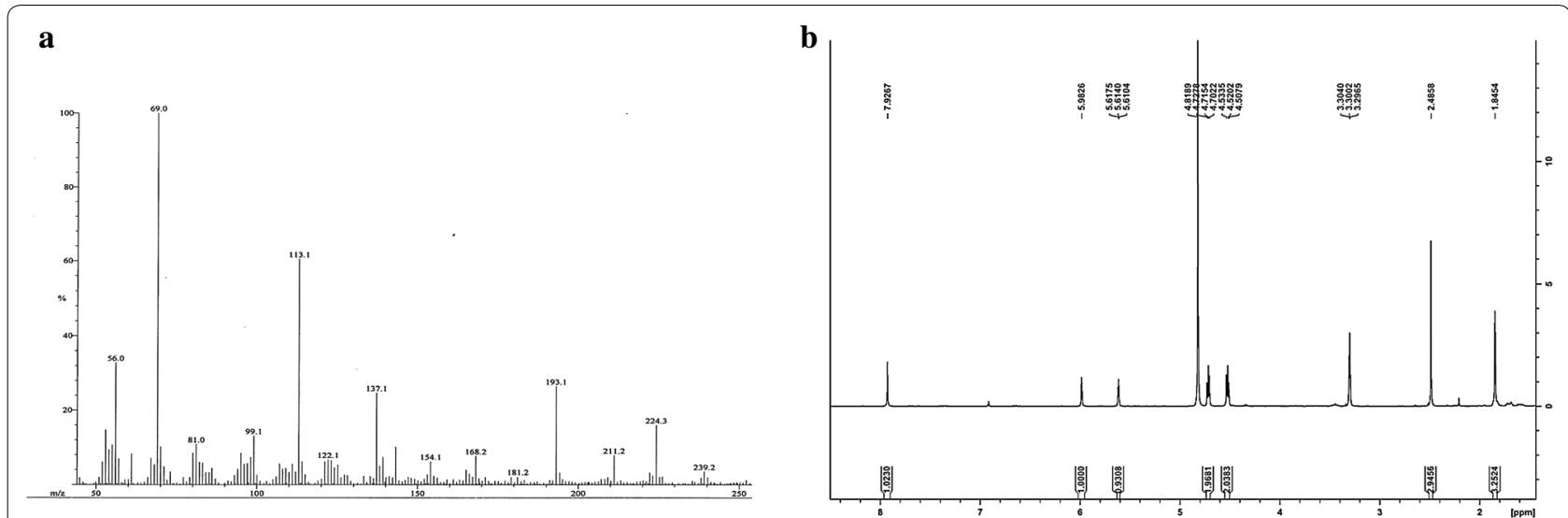

Fig. 2 a El-MS spectra of synthesized compound 1, b ${ }^{1} \mathrm{H}$ NMR spectra of synthesized compound 1 

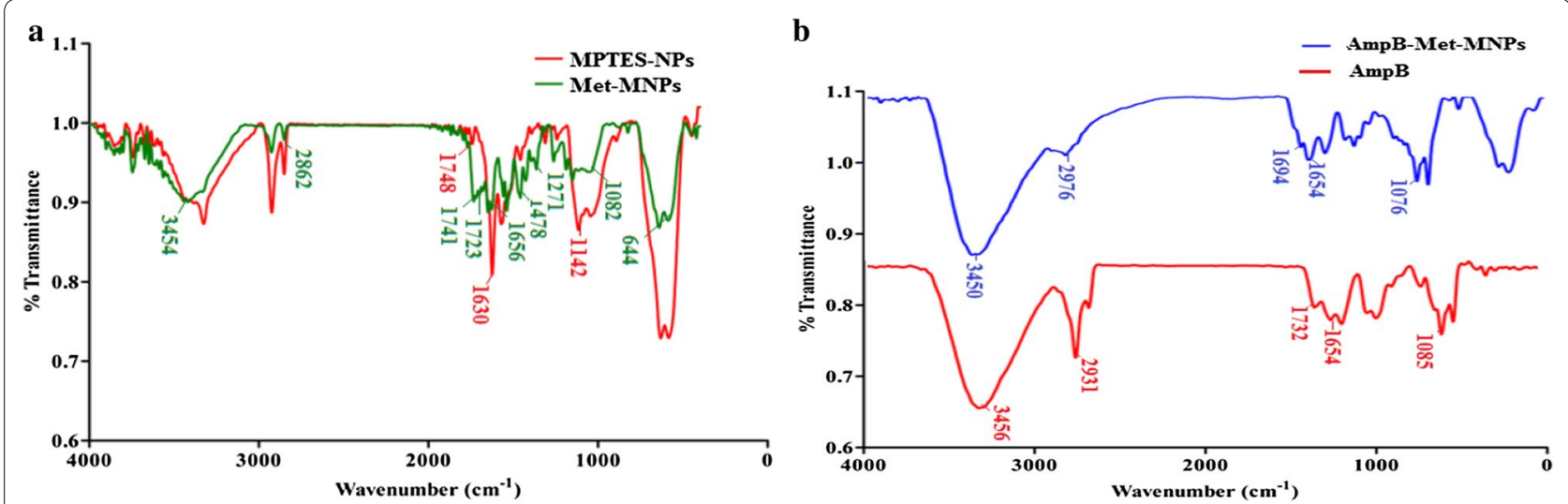

Fig. 3 Comparative FT-IR spectra of a MPTES-NPs with Met-MNPs. b AmpB-Met-MNPs along with AmpB showing the formation of AmpB-Met-MNPs

The prepared MPTES modified NPs show $\mathrm{CH}_{2}$ bands at $2862 / \mathrm{cm}$, for the propyl chain and the presence of $(\mathrm{C}=\mathrm{O})$ was associated with the band at $1748 / \mathrm{cm}$. Furthermore, band at $1630 / \mathrm{cm}$ corresponds to $(\mathrm{C}=\mathrm{C})$ stretch (Ireland et al. 2006; Kawish et al. 2020). Met modified NPs shows two stretching frequencies at $1741 / \mathrm{cm}$ and $1728 /$ $\mathrm{cm}$ corresponds to two ester $(\mathrm{C}=\mathrm{O})$. In addition, symmetric $(C=N)$ aromatic stretch was observed at $1656 / \mathrm{cm}$ corresponds to imidazole ring. Moreover, peak at 644/ $\mathrm{cm}$ on Met-MNPs evidenced that Met is grafted on the surface of MNPs. Amphotericin B reveals characteristic absorption around $1733 / \mathrm{cm}$ and $1654 / \mathrm{cm}$ corresponding to $(\mathrm{C}=\mathrm{O})$ and $(\mathrm{C}=\mathrm{C})$ moieties (Jabri et al. 2018). Stretching frequency at $3456 / \mathrm{cm}$ corresponds to $\mathrm{OH}$ stretching. Amp B-Met-MNPs nanoparticles show slight variation in absorption frequencies -the peak at $1732 / \mathrm{cm}$ of carboxylic acid $(\mathrm{C}=\mathrm{O})$ was shifted to $1694 / \mathrm{cm}$ and the peak at $1654 / \mathrm{cm}$ remained unchanged. Frequency at 1085/ $\mathrm{cm}$ of acetal bond was shifted to $1076 \mathrm{~cm}^{-1}$ (Fig. 3b). The absorption at $3456 / \mathrm{cm}$ of $\mathrm{OH}$ was shifted to $3450 /$ $\mathrm{cm}$ which may be attributed to increased chelation of hydroxyl groups with MNPs and secondary hydrogen bonding and $\pi-\pi$ stacking interaction in between drug and the synthesized NPs.

Particle size and colloidal stability are considered key factors for biological applications (De Jong and Borm
2008). Generally, magnetic nanoparticles are infamous for protein aggregation when introduced in a protein rich media due to their large surface to volume ratio. This undesirable characteristic restricts the biological accumulation of MNPs as biological uptake is mainly dependent upon the colloidal stability of NPs (Oh et al. 2017). The mean hydrodynamic diameter of Met-MNPs and Met-MNPs-Amp was found to be $193.13 \pm 6.8 \mathrm{~nm}$ and $218.53 \pm 13.4 \mathrm{~nm}$ (Table 1), respectively. Moreover the PDI values were $0.17 \pm 0.05$ and $0.26 \pm 0.01$, respectively. The zeta potential of Met-MNPs and Met-MNPsAmp was found to be $-13.7 \pm 0.9$ and $-18.6 \pm 4.5 \mathrm{mV}$ (Table 1). The increased size and zeta potential values of Met-MNPs-Amp gives an evidence of drug adsorption on Met-MNPs. Furthermore, aggregation is less likely to occur with high zeta potential due to increased electrostatic repulsion which gives long term stability of our developed NPs. The vacant Met-MNPs nanoparticles were nearly spherical in morphology as revealed by AFM (Fig. 4a). Whereas the amphotericin B loaded Met-MNPs-Amp nanoparticles shows slight distortion in morphology which may be an evidence of successive entrapment of drug onto nanoparticles (Fig. 4b).

The characteristic property of any drug carrier is the ability to carry an amount of drug with it, which shows the overall therapeutic outcomes. High therapeutic

Table 1 Size, zeta potential, PDI, drug entrapment efficiency at several ratios of Met-MNPs nanoparticles

\begin{tabular}{llcccc}
\hline Nanoparticles & Ratios (drug:NPs) & Size $(\mathbf{n m})$ & PDI & Zeta potential (mV) & $\begin{array}{l}\text { Entrapment } \\
\text { efficiency } \\
\mathbf{( \% )}\end{array}$ \\
\hline Met-MNPs & None & $193.13 \pm 6.8$ & $0.17 \pm 0.05$ & $-13.7 \pm 0.9$ & None \\
AmpB-Met-MNPs & $1: 1$ & $218.53 \pm 13.4$ & $0.26 \pm 0.01$ & $-18.6 \pm 4.5$ & $77.05 \pm 2.04$ \\
AmpB-Met-MNPs & $2: 1$ & $658.5 \pm 510$ & $0.65 \pm 0.3$ & $-17.2 \pm 14$ & $68.42 \pm 3.21$ \\
AmpB-Met-MNPs & $3: 1$ & $1419 \pm 189$ & 1 & $-9.43 \pm 3.2$ & $52.93 \pm 5.32$ \\
\hline
\end{tabular}



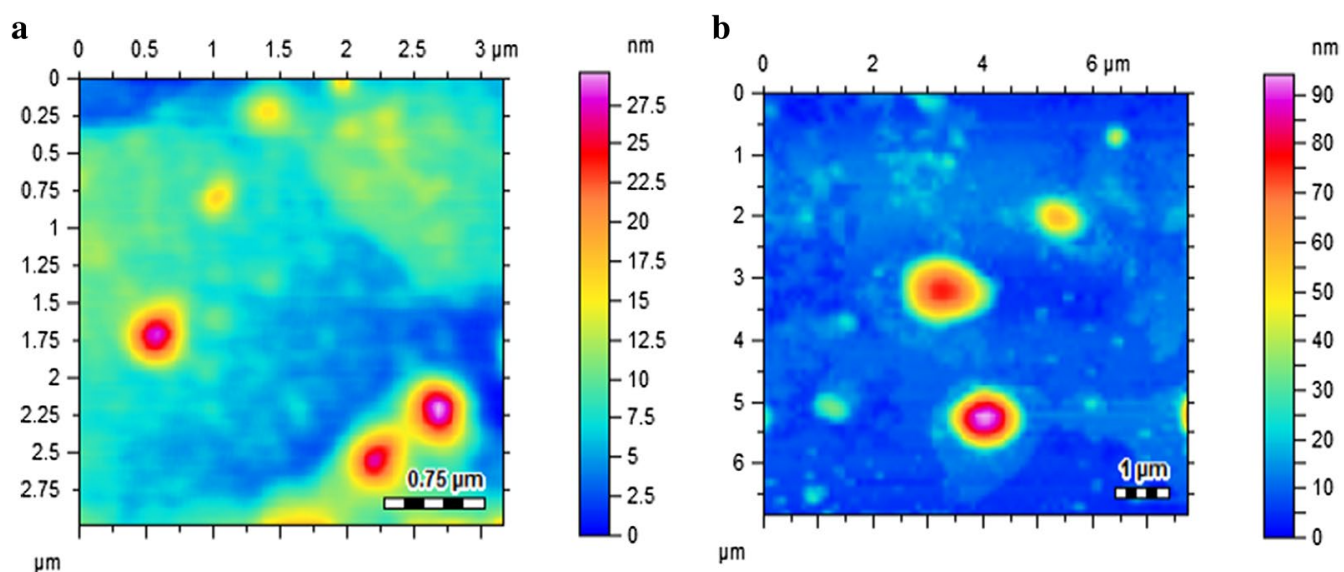

Fig. 4 Atomic force microscopic images of $\mathbf{a}$ Met-MNPs and $\mathbf{b}$ AmpB-Met-MNPs showing nearly spherical morphology

loading is essential for maintaining prolonged therapeutic effect at the site of action which minimizes the risk of over dosage and its linked toxicities (Imran et al. 2016a, b). Our developed Met-MNPs formulation undergo various blending with $\mathrm{AmpB}$ to obtain a formulation containing high amounts of the drug. Interestingly MetMNPs entrapped $77.05 \pm 2.04 \%$ of drug at 1:1 ratio excellent colloidal stability as depicted in (Table 1). The higher drug entrapment may be attributed to increased chelation in the form of hydroxyl groups on AmpB, $\pi-\pi$
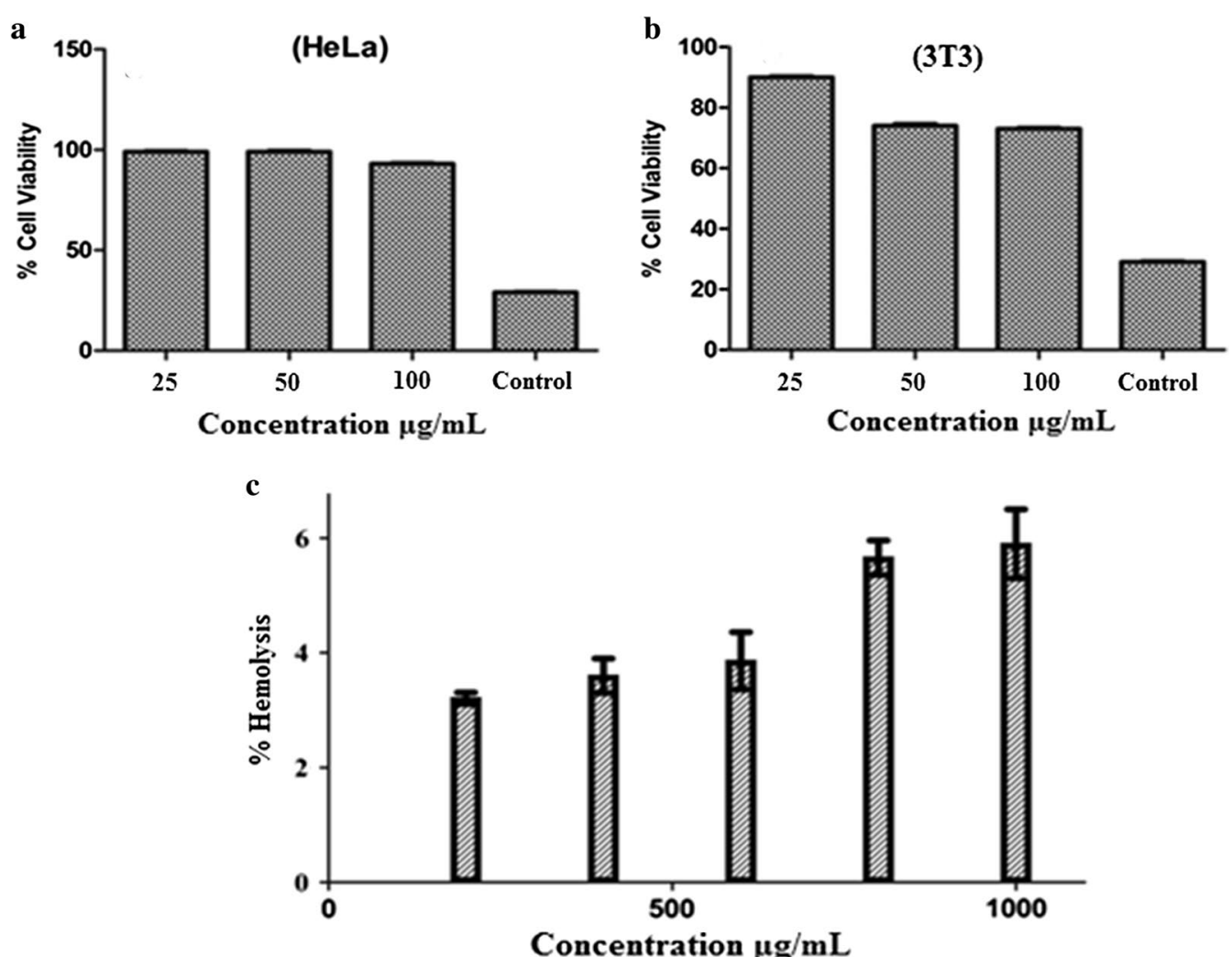

Fig. 5 a Cell viability profile of Met-MNPs against Hela cell lines. b Cell viability profile of developed Met-MNPs against 3T3-NIH cell lines. c Concentration dependent hemolytic activity of synthesized Met-MNPs against RBCs 
stacking and hydrogen bonding interactions between drug molecules and Met-MNPs.

The most predominant factor for any drug delivery excipient is its biocompatibility Therefore, prior to in vivo applications, biocompatibility is considered an essential factor (Fischer et al. 2003). Mouse embryonic fibroblast 3T3 and human cervical cell lines HeLa are commonly used cell lines showing reproducibility. 3T3 and HeLa cell lines were exposed to Met-MNPs-Amp for the evaluation of cytotoxicity using MTT assay. For comparison, doxorubicin and cycloheximide were used as positive controls. Met-MNPs-Amp was incubated at various concentrations against $3 \mathrm{~T} 3$ and HeLa cell lines. These nanoparticles revealed cell viability in a concentration dependent manner. Experiments conducted against HeLa cell lines, showed that the cell viability was found to be $96 \pm 0.65 \%$ at concentration of $100 \mu \mathrm{g} / \mathrm{mL}$, whereas, $3 \mathrm{~T} 3$ cell lines showed cell viability about $74 \pm 1.93 \%$ after $48 \mathrm{~h}$ as depicted in Fig. 5a, b, respectively. This study reveals that the synthesized nanoparticles are biocompatible. Surface functionalization with biocompatible molecules not only lowers toxicity but enables them the carry drug cargo with it.
The interaction of surface functionalized magnetic NPs with negatively charged membranes have been studied via hemolysis study. The release of hemoglobin from cells determines the membrane destruction characteristics of NPs (Fischer et al. 2003). Titron X-100 was used as $100 \%$ values for erythrocytes in this study. Met-MNPs-Amp were used at different concentrations ranging from 200, 400, 600, 800 and $1000 \mu \mathrm{g} / \mathrm{mL}$ and released hemoglobin was quantitatively analyzed at $541 \mathrm{~nm}$ (Fig. 5c). MetMNPs-Amp showed less than $10 \%$ hemolytic activity even at a higher concentration of $1 \mathrm{mg} / \mathrm{mL}$ indicating the membrane friendly properties (i.e. did not cause any disruption to biological membrane).

Met-MNPs-Amp demonstrated dose dependent amoebicidal effects from 100 to $6.25 \mu \mathrm{g} / \mathrm{mL}$ (Fig. 6a). These findings suggest that nanoparticles are valuable in developing future treatments against these deadly infections (Anwar et al. 2018). The $\mathrm{IC}_{50}$ was found to be $50 \mu \mathrm{g} /$ $\mathrm{mL}$ against $A$. castellanii trophozoites. Met-MNPsAmp showed significantly improved amoebicidal effects as compared to all respective controls at both 100 and $50 \mu \mathrm{g} / \mathrm{mL}$ (Fig. 6b, c). Met-MNPs-Amp elicited 66\% and $55 \%$ A. castellanii trophozoite death at 100 and $50 \mu \mathrm{g} /$ $\mathrm{mL}$, respectively. The $\mathrm{IC}_{50}$ against $A$. castellanii cysts was
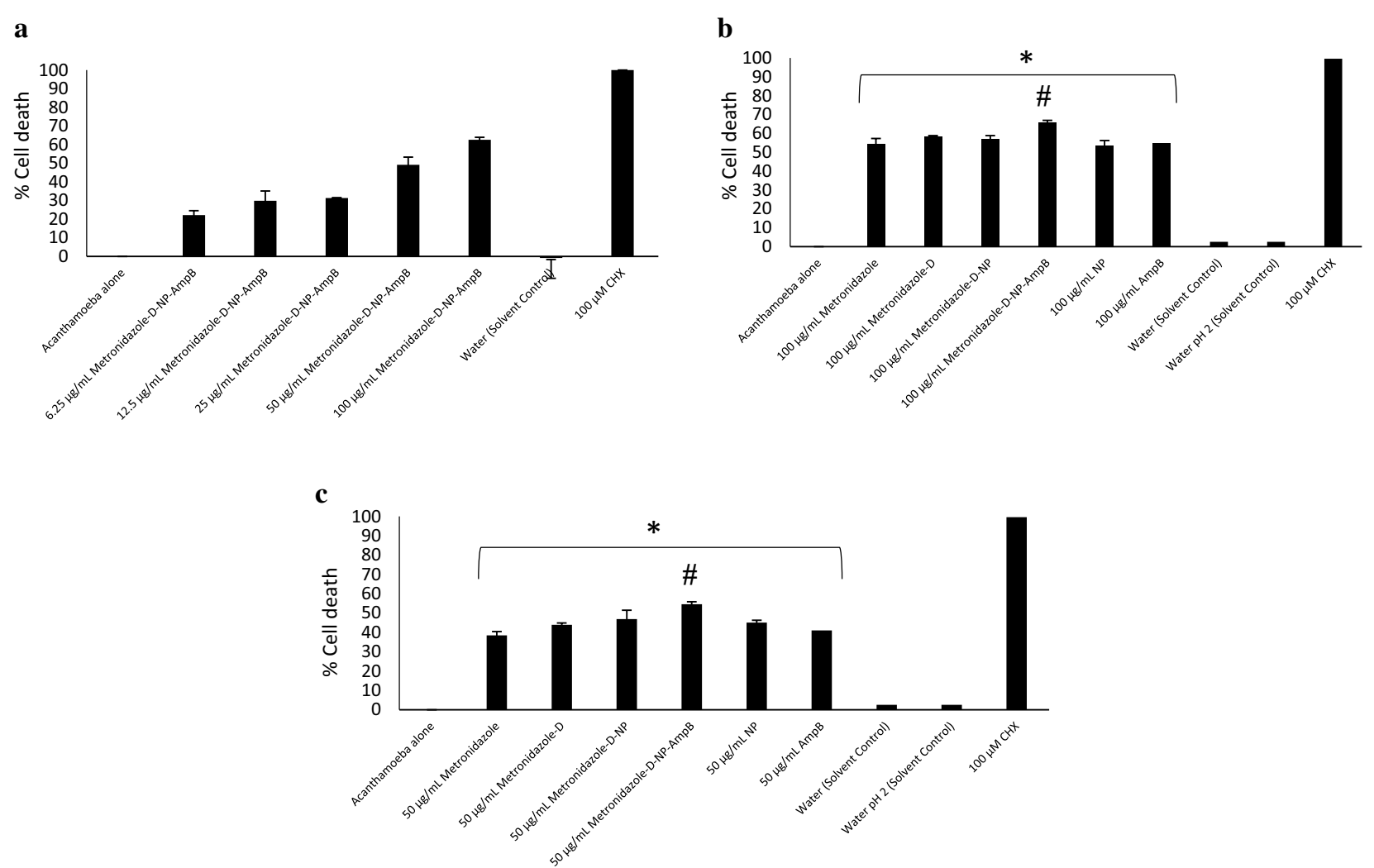

Fig. 6 a $I_{50}$ of AmpB-Met-MNPs against trophozoites. b Antiamoebic activity against Acanthamoeba castellanii trophozoites at $100 \mu \mathrm{gg} / \mathrm{mL}$. c Antiamoebic activity against Acanthamoeba castellanii trophozoites at $50 \mu \mathrm{g} / \mathrm{mL}\left({ }^{*} \mathrm{P}<0.05\right.$ as compared to negative control) 
found to be $50 \mu \mathrm{g} / \mathrm{mL}$ (Fig. 7a). Met-MNPs-Amp caused $61 \%$ cyst death at $100 \mu \mathrm{g} / \mathrm{mL}$ and $48 \%$ cyst death at $50 \mu \mathrm{g} /$ $\mathrm{mL}$ (Fig. 7b, c). A significant improvement in the cysticidal activity of Met-MNPs-Amp was seen when compared to metronidazole alone, Met-NPs, as well as NPs alone at both100 and $50 \mu \mathrm{g} / \mathrm{mL}$ (Fig. 7b, c).

\section{Discussion}

Given that the mortality rate of infection due to brain eating amoebae is over $90 \%$, we tested the aforementioned conjugated drug. Met-MNPs-Amp demonstrated dose dependent amoebicidal effects from 100 to $6.25 \mu \mathrm{g} /$ $\mathrm{mL}$ (Fig. 6a). These findings suggest that nanoparticles are valuable in developing future treatments against these deadly infections (Anwar et al. 2018). These findings are not surprising as metronidazole is a common antiprotozoal drug used against Giardia and Entamoeba and Trichomonas (Freeman et al. 1997). It has also been the part of regimen used to treat cases of primary amoebic meningoencephalitis (PAM) (Gupta et al. 2015; Deetz et al. 2003). Metronidazole is also known to inhibit the nucleic acid synthesis of brain-eating amoebae (Mungroo et al. 2019). However, it has shown limited efficacy against the later stages of infection and has been replaced with other drugs (Deetz et al. 2003). On the other hand, amphotericin B has been the corner stone of therapy against $N$. fowleri and $B$. mandrillaris by targeting ergosterol and disrupting membrane (Grace et al. 2015). Albeit its high associated toxicity at the higher dosage required to kill amoebae, the administration of this macrolide is an issue which has been addressed by its incorporation with nanomaterials. Amphotericin B conjugated with gold and silver nanoparticles have shown enhanced antiamoebic effects against $A$. castellanii and $N$. fowleri (Anwar et al. 2019b; Rajendran et al. 2017). In another report, Lemke et al. reported the increased drug delivery of amphotericin B in the form of nanosuspension to the brain against Balamuthia infection (Lemke et al. 2010). Since metronidazole, and amphotericin B have been successfully used clinically against brain-eating amoebae infections, we successfully formulated novel drug delivery nanoparticles containing both of these drugs. For this purpose, iron oxide nanoparticles were used as carriers for the first time against these amoebae. Magnetic iron oxide nanoparticles were chemically conjugated with metronidazole which were further loaded with amphotericin B to exhibit synergistic effects. The small size of these nanoparticles ensures the targeted delivery of metronidazole and amphotericin $B$ to their respective active sites in brain. These nanoparticles are also expected to increase the sensitivity of MRI for the detection of amoebae in the brain. Furthermore, the magnetic nature of

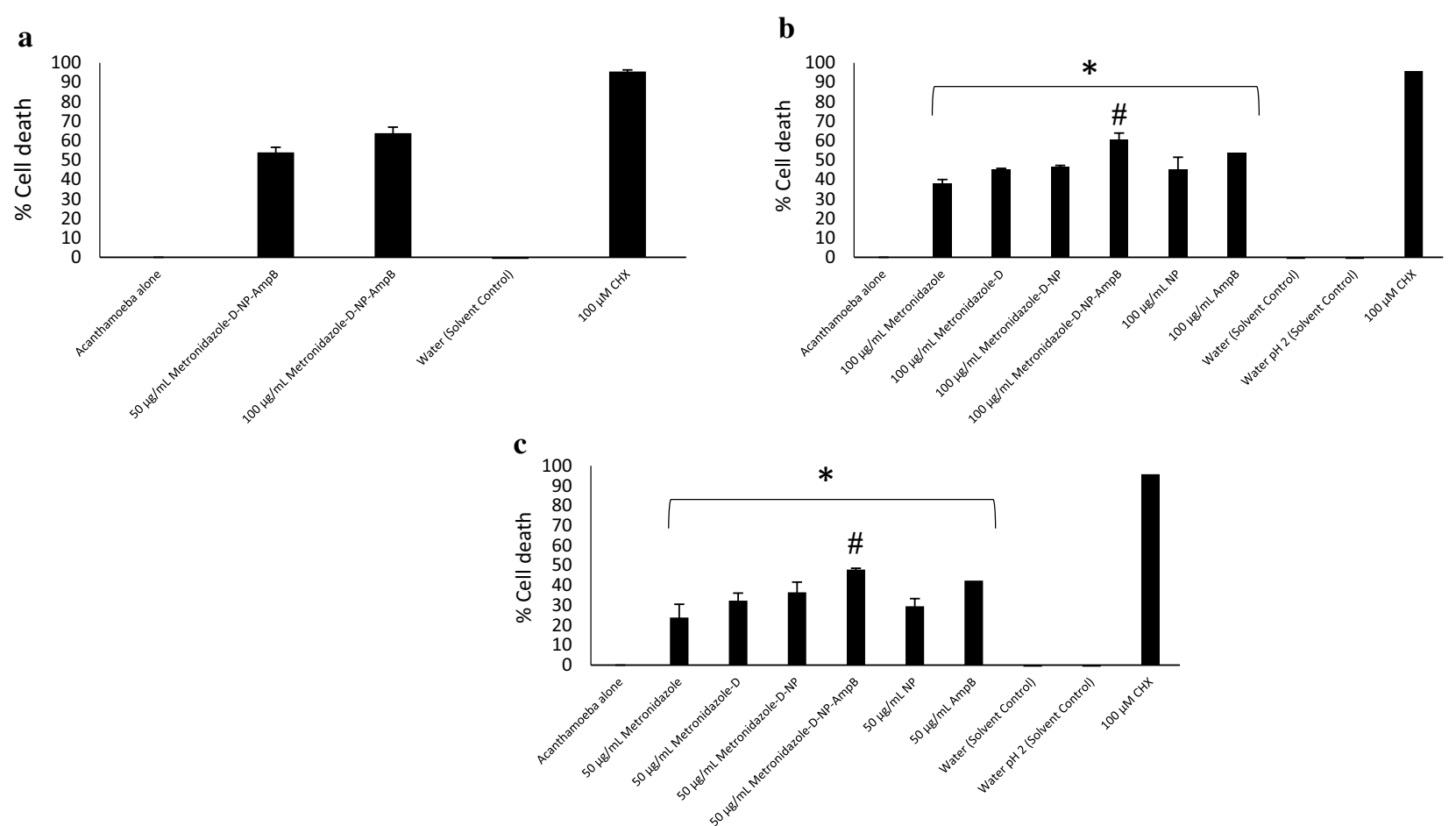

Fig. 7 a $I C_{50}$ of AmpB-Met-MNPs against cysts. b Antiamoebic activity against Acanthamoeba castellanii cysts at $100 \mu \mathrm{g} / \mathrm{mL}$. c Antiamoebic activity against Acanthamoeba castellanii cysts at $50 \mu \mathrm{g} / \mathrm{mL}$ (\#P<0.05 as compared to drugs and nanoparticles alone) 
these nanoparticles can be utilized to isolate amoebae from biological and environmental samples which can be useful platforms for biomedical applications.

In summary, metronidazole conjugated iron oxide nanoparticles loaded with amphotericin B were synthesized. These nanoparticles were thoroughly characterized by FT-IR, AFM, and DLS analyses. These nanoparticles were found to be biocompatible when tested against human and rat cell lines, and also did not show haemolytic activity in vitro. These nanoparticles were designed to exhibit potent antiamoebic effects against the pathogenic parasite $A$. castellanii. This study showcased the antiamoebic activity of magnetic iron oxide nanoparticles for the first time against freeliving amoebae. The results from amoebicidal and cysticidal studies against $A$. castellanii showed that these nanoparticles hold potential for future mode of action and in vivo studies.

\section{Acknowledgements}

The work in this paper was supported, in part, by the Open Access Program from the American University of Sharjah. This paper represents the opinions of the author(s) and does not mean to represent the position or opinions of the American University of Sharjah.

\section{Authors' contributions}

AA and NAK conceived the project and obtained funding. KI and AA prepared the materials, conjugated drugs under the supervision of MRS. RS and NAK helped design experimental plans. SAOA carried out all experiments under the supervision of AA and RS. SAOA and AA wrote the first draft of the manuscript. All authors approved the final manuscript.

\section{Funding}

This work is funded by Sunway University Malaysia, GRTIN-RRO-98-2020 and American University of Sharjah.

\section{Data availability}

Data will be provided upon request on a case to case basis.

\section{Ethics approval and consent to participate}

The ethics approval for human participants was obtained from the International Center for Chemical and Biological Sciences Independent Ethics Committee and approval code is: ICCBS/IEC/LET/015/2018. All methods were carried out in accordance with relevant guidelines and regulations.

\section{Consent for publication}

Not applicable.

\section{Competing interests}

The authors declare that they have no competing interests.

\footnotetext{
Author details

${ }^{1}$ Department of Biological Sciences, School of Science and Technology, Sunway University, Subang Jaya, 47500 Selangor, Malaysia. ${ }^{2}$ HEJ Research Institute of Chemistry, International Center for Chemical and Biological Sciences, University of Karachi, Karachi 75270, Pakistan. ${ }^{3}$ Faculty of Defence Science and Technology, National Defence University of Malaysia, 57000 Kuala Lumpur, Malaysia. ${ }^{4}$ Department of Biology, Chemistry and Environmental Sciences, College of Arts and Sciences, American University of Sharjah, Sharjah 26666, United Arab Emirates.
}

Received: 29 April 2020 Accepted: 6 July 2020

Published online: 17 July 2020

\section{References}

Ahmed D, Anwar A, Khan AK, Ahmed A, Shah MR, Khan NA (2017) Size selectivity in antibiofilm activity of 3-(diphenylphosphino) propanoic acid coated gold nanomaterials against Gram-positive Staphylococcus aureus and Streptococcus mutans. AMB Express 7:1-11

Ali M, Jamal SB, Farhat SM (2020) Naegleria fowleri in Pakistan. Lancet Infect Dis 20:27-28

Anwar A, Siddiqui R, Khan NA (2018) Importance of theranostics in rare braineating amoebae infections. ACS chem Neurosci 10:6-12

Anwar A, Chi Fung L, Anwar A, Jagadish P, Numan A, Khalid M, Shahabuddin S, Siddiqui R, Khan NA (2019a) Effects of shape and size of cobalt phosphate nanoparticles against Acanthamoeba castellanii. Pathogens 8:260

Anwar A, Masri A, Rao K, Rajendran K, Khan NA, Shah MR, Siddiqui R (2019b) Antimicrobial activities of green synthesized gums-stabilized nanoparticles loaded with flavonoids. Sci Rep 9:3122

Anwar A, Siddiqui R, Shah MR, Khan NA (2019c) Gold nanoparticles conjugation enhances antiacanthamoebic properties of nystatin, fluconazole and amphotericin B. J Microbiol Biotechnol 29:171-177

Azam A, Ahmed AS, Oves M, Khan MS, Habib SS, Memic A (2012) Antimicrobial activity of metal oxide nanoparticles against Gram-positive and Gramnegative bacteria: a comparative study. Int J Nanomed 7:6003

Cope JR (2013) Investigational drug available directly from CDC for the treatment of infections with free-living amebae. Morb Mortal Wkly Rep 62(33):666

De Jong WH, Borm PJ (2008) Drug delivery and nanoparticles: applications and hazards. Int J Nanomed 3:133

Deetz TR, Sawyer MH, Billman G, Schuster FL, Visvesvara GS (2003) Successful treatment of Balamuthia amoebic encephalitis: presentation of 2 cases. Clin Infect Dis 37:1304-1312

Fischer D, Li Y, Ahlemeyer B, Krieglstein J, Kissel T (2003) In vitro cytotoxicity testing of polycations: influence of polymer structure on cell viability and hemolysis. Biomaterials 24:1121-1131

Freeman CD, Klutman NE, Lamp KC (1997) Metronidazole. Drugs 54:679-708

Gomart G, Denis J, Bourcier T, Dory A, Abou-Bacar A, Candolfi E, Sauer A (2018) In vitro amoebicidal activity of Titanium dioxide/UV-A combination against Acanthamoeba. Invest Ophthalmol Vis Sci 59:4567-4571

Grace E, Asbill S, Virga K (2015) Naegleria fowleri: pathogenesis, diagnosis, and treatment options. Antimicrob Agents Chemother 59:6677-6681

Gupta R, Parashar M, Kale A (2015) Primary amoebic meningoencephalitis. J Assoc Physicians India 63

Hoseinzadeh E, Makhdoumi P, Taha P, Hossini H, Stelling J, Amjad Kamal M (2017) A review on nano-antimicrobials: metal nanoparticles, methods and mechanisms. Curr Drug Metab 18:120-128

Imran M, Muazzam AG, Habib A, Matin A (2016a) Synthesis, characterization and amoebicidal potential of locally synthesized TiO2 nanoparticles against pathogenic Acanthamoeba trophozoites in vitro. J Photochem Photobiol B 159:125-132

Imran M, Shah MR, Ullah F, Ullah S, Elhissi AM, Nawaz W, Ahmad F, Sadiq A, Ali I (2016b) Sugar-based novel niosomal nanocarrier system for enhanced oral bioavailability of levofloxacin. Drug Delivery 23:3653-3664

Ireland DC, Colgrave ML, Craik DJ (2006) A novel suite of cyclotides from Viola odorata: sequence variation and the implications for structure, function and stability. Biochem J 400:1-12

Jabri T, Imran M, Rao K, Ali I, Arfan M, Shah MR (2018) Fabrication of lecithingum tragacanth muco-adhesive hybrid nano-carrier system for in vivo performance of amphotericin B. Carbohydr Polym 194:89-96

Kawish M, Elhissi A, Jabri T, Iqbal KM, Zahid H, Shah MR (2020) Enhancement in oral absorption of ceftriaxone by highly functionalized magnetic iron oxide nanoparticles. Pharmaceutics 12:492

Lemke A, Kiderlen AF, Petri B, Kayser O (2010) Delivery of amphotericin B nanosuspensions to the brain and determination of activity against Balamuthia mandrillaris amebas. Nanomed Nanotechnol Biol Med 6:597-603

Manatunga DC, de Silva RM, de Silva KN, de Silva N, Bhandari S, Yap YK, Costha NP (2017) pH responsive controlled release of anti-cancer hydrophobic drugs from sodium alginate and hydroxyapatite bi-coated iron oxide nanoparticles. Eur J Pharm Biopharm 117:29-38

Mungroo MR, Anwar A, Khan NA, Siddiqui R (2019) Brain-eating amoebae infection: challenges and opportunities in chemotherapy. Mini Rev Med Chem 19:980-987

Oh Y, Je J-Y, Moorthy MS, Seo H, Cho WH (2017) pH and NIR-light-responsive magnetic iron oxide nanoparticles for mitochondria-mediated apoptotic 
cell death induced by chemo-photothermal therapy. Int J Pharm 531:1-13

Petcharoen K, Sirivat A (2012) Synthesis and characterization of magnetite nanoparticles via the chemical co-precipitation method. Mater Sci Eng B $177: 421-427$

Raghunath A, Perumal E (2017) Metal oxide nanoparticles as antimicrobial agents: a promise for the future. Int J Antimicrob Agents 49:137-152

Rajendran K, Anwar A, Khan NA, Siddiqui R (2017) Brain-eating amoebae: silver nanoparticle conjugation enhanced efficacy of anti-amoebic drugs against Naegleria fowleri. ACS Chem Neurosci 8:2626-2630

RajendranK Anwar A, Khan NA, AslamZ Raza M, Siddiqui R (2019) Oleic acid coated silver nanoparticles showed better in vitro amoebicidal effects against Naegleria fowleri than amphotericin B. ACS Chem Neurosci. https ://doi.org/10.1021/acschemneuro.9b00289

Saif B, Wang C, Chuan D, Shuang S (2015) Synthesis and characterization of $\mathrm{Fe}_{3} \mathrm{O}_{4}$ coated on APTES as carriers for morin-anticancer drug. J Biomater Nanobiotechnol 6:267

Schuster FL, Visvesvara GS (2004) Free-living amoebae as opportunistic and non-opportunistic pathogens of humans and animals. Int J Parasitol 34:1001-1027
Siddiqui R, Khan NA (2014) Primary amoebic meningoencephalitis caused by Naegleria fowleri: an old enemy presenting new challenges. PLoS Neglect Trop Dis 8:e3017

Vimbela GV, Ngo SM, Fraze C, Yang L, Stout DA (2017) Antibacterial properties and toxicity from metallic nanomaterials. Int J Nanomed 12:3941

Visvesvara GS (2013) Infections with free-living amebae. In: Handbook of clinical neurology. vol 114. Elsevier, pp 153-168

Visvesvara GS, Moura H, Schuster FL (2007) Pathogenic and opportunistic freeliving amoebae: Acanthamoeba spp., Balamuthia mandrillaris, Naegleria fowleri, and Sappinia diploidea. FEMS Immunol Med Microbiol 50:1-26

Wang L, Hu C, Shao L (2017) The antimicrobial activity of nanoparticles: present situation and prospects for the future. Int J Nanomed 12:1227

\section{Publisher's Note}

Springer Nature remains neutral with regard to jurisdictional claims in published maps and institutional affiliations.

\section{Submit your manuscript to a SpringerOpen ${ }^{\circ}$ journal and benefit from:}

- Convenient online submission

- Rigorous peer review

- Open access: articles freely available online

- High visibility within the field

- Retaining the copyright to your article

Submit your next manuscript at $\boldsymbol{\nabla}$ springeropen.com 